\title{
Effective use of the Pig-a gene mutation assay for mutagenicity screening: measuring CD59-deficient red blood cells in rats treated with genotoxic chemicals
}

\author{
Takafumi Kimoto, Satsuki Chikura, Kumiko Suzuki-Okada, Xiao-mei Kobayashi, \\ Yasuhiro Itano, Daishiro Miura and Yoshinori Kasahara
}

TEIJIN Pharma Limited, 4-3-2, Asahigaoka, Hino Tokyo 191-8512, Japan

(Received June 10, 2012; Accepted July 11, 2012)

\begin{abstract}
The Pig- $a$ gene mutation assay using perpherial blood erythrocytes is being investigated as a screening tool for assessing mutagenicity in vivo. In this study, we evaluated two distinct approaches for performing the Pig- $a$ assay in rats. We used antibodies to CD45 or the erythroid marker HIS49 to identify red blood cells (RBCs), and then monitored the kinetics of Pig- $a$ mutant frequency, as measured by the frequency of CD59-deficient RBCs, in rats treated with the genotoxic chemicals, $N$-ethyl$N$-nitrosourea, cyclophosphamide, 4-nitroquinoline-1-oxide, and ethylmethanesulfonate. In some instances, micronucleus frequency also was measured in the same animals. Time- and dose-related increases in Pig-a mutant frequency were found in all the chemical-treated groups, except for the groups treated with cyclophosphamide, which was a potent inducer of micronuclei. The two different approaches we employed were comparable for measuring induced mutant frequencies, but our historical data showed that the mean background frequencies for the CD45/CD59 method and the HIS49/CD59 method were $12.7 \times 10^{-6}$ and $5.5 \times 10^{-6}$, respectively. The relatively low, stable background mutant frequency associated with the HIS49/CD59 method indicates that it may have greater power for discriminating weak induced responses. These results suggest that the HIS49/CD59 method is a promising tool for measuring Pig-a mutant RBCs. In addition, differences in their manifestation kinetics and in their relative sensitivity for detecting different test compounds suggest that the combination of the Pig-a assay and the micronucleus assay may be effective in identifying in vivo genotoxicity.
\end{abstract}

Key words: In vivo gene mutation assay, In vivo micronucleus assay,

Phosphatidylinositol glycan complementation class A gene, Flow cytometry,

Peripheral red blood cells

\section{INTRODUCTION}

Chemicals that induce genetic damage to DNA (e.g., gene mutation and chromosome aberration) may cause cancer and/or heritable defects (Duesberg et al., 2005). Both in vitro and in vivo genotoxicity tests have been established to assess the genotoxicological risk of chemicals, including candidate pharmaceuticals (Kirkland et al., 2005; Rothfuss et al., 2011). In vivo tests fall into three general categories, those that measure clastogenicity and possibly aneugenicity, those that measure gene mutation, and those that measure DNA damage. The micronucleus test, which can evaluate the ability of chemicals to induce structural and numerical chromosome aberration, is the primary in vivo genotoxicity test used in regulatory science (Tweats et al., 2007). In particular, remarkable developments have been made in flow-cytometric scoring of micronucleated reticulocytes (MN-RET) in peripheral blood (PB) as a screening assay (Dertinger et al., 1996, 2011a). The Comet assay, which can evaluate the ability of chemicals to induce primary DNA damage, has a validated protocol (Hartmann et al., 2003), and is undergoing Organization for Economic Co-operation and Development (OECD) Test Guideline development. It is cited as a second in vivo test by the International Conference on Harmonization (ICH) S2(R1) guideline for further assessing the in vivo genotoxicity of human drugs (ICHS2(R1), Current step 4 version dated

Correspondence: Takafumi Kimoto (E-mail: t.kimoto@teijin.co.jp) 
9 November 2011). The gene mutation assays conducted with transgenic animals and the Hypoxanthine phosphoribosyltransferase gene (Hprt) lymphocyte assay have been used worldwide to assess the risk of in vivo gene mutation (Casciano et al., 1999; Thybaud et al., 2003). However, these tests are considered impractical for routine use and no in vivo gene mutation assays are included in the ICH S2(R1) battery.

In recent years, a novel in vivo gene mutation assay has been developed that uses the phosphatidylinositol glycan, class A gene (Pig- $a$ in rodents, $P I G-A$ in humans) as an endogenous reporter (Dertinger and Heflich, 2011; Dobrovolsky et al., 2010a; Peruzzi et al., 2010). The principle of the assay is that the pathway for glycosylphosphatidylinositol (GPI) anchor synthesis requires the Pig- $a$ gene product; thus the absence of GPI-anchored proteins on the surface of mammalian cells (usually erythrocytes) can be used as a reporter of Pig- $a$ mutation. The Pig-a assay has several advantages that recommend it as a prediction tool for in vivo somatic mutation. One is that results can be generated in only hours using flow cytometry, with no cell culture necessary. Another is that the Pig- $a$ assay can be used for measuring mutation in various mammals, including rodents and humans, because the gene is highly conserved (Kawagoe et al., 1994; Phonethepswath et al., 2008; Takeda et al., 1993). While the assays that measure mutation in rodent erythrocytes have benefited from the most developmental effort, technical trials of the red blood cell (RBC) $P I G-A$ assay in humans and in non-human primates have demonstrated their feasibility for measuring mutation in these species (Dobrovolsky et al., 2009, 2011).

Previous studies have progressively clarified the characteristics of the Pig- $a$ assay. During our efforts to develop a flow cytometric Pig- $a$ assay for measuring gene mutation in $N$-ethyl- $N$-nitrosourea (ENU) treated rodents, we demonstrated increased frequencies of GPI-anchored-proteindeficient peripheral RBCs, bone marrow erythroids, and splenocytes (Kimoto et al., 2011a, 2011b; Miura et al., 2008a, 2008b). The use of non-nucleated RBCs to measure Pig- $a$ gene mutation has been supported by sequence analysis of the Pig- $a$ gene in GPI-anchored-protein-deficient bone marrow erythroids, which are the presumed precursors of PB RBCs (Kimoto et al., 2011b). Related to this, we have proposed a model for Pig- $a$ mutant manifestation to account for the accumulation and persistence of GPI-deficient Pig- $a$ mutant RBCs observed in ENU treated rats (Miura et al., 2009). The Pig- $a$ assay can be performed with microliter volumes of PB so that the integration of the assay into repeated-dose toxicology studies or other genetic toxicology studies is feasible, which is con- sistent with spirit of the 'three Rs' (Dertinger et al., 2010; Phonethepswath et al., 2010). In addition, the methodology for measuring Pig-a mutant cells developed by Dertinger and his colleagues was found to be reproducible and transferable in an international Pig-a collaborative study (Dertinger et al., 2011c). These dedicated efforts raise hope that the Pig- $a$ assay may be an effective tool for evaluating in vivo mutagenicity.

There are various approaches for measuring RBC Pig-a mutant frequency in rats. Miura et al. (2008a) developed an assay that labels PB with fluorescent antibodies specific for the leukocyte common antigen, CD45, to exclude leukocytes from the analysis. Another approach is to first leukodeplete PB samples by gradient centrifugation and then stain for reticulocytes (RETs) with Thiazole Orange or SYTO 13, which binds nucleic acid (Bryce et al., 2008; Phonethepswath et al., 2008). This approach was used in the international collaborative study and made it possible to distinguish RETs from mature RBCs and to evaluate Pig-a mutant frequencies in both populations at the same time (Dertinger et al., 2011c). While both scoring approaches described above excluded nucleated cells from the analysis based on leukocyteassociated fluorescence (i.e., anti-CD45 or high nucleic acid dye-associated signal), the approach developed by Dobrovolsky et al. (2010b) used the anti-rat erythroid marker, HIS49, to positively identify RBCs in PB. Enriching the erythroid population with HIS49 antibody facilitates performing the Pig- $a$ assay as it evaluates Pig$a$ mutant frequency specifically in RBCs without the need to leukodeplete the blood. However, most of the available Pig- $a$ mutant data has been produced using leukodepletion, and little is known about the relative merits of performing the RBC Pig- $a$ assay using anti-CD45 or HIS49 antibodies.

In this study, we evaluated the performance of two distinct Pig- $a$ assays, hereafter referred as the CD45/ CD59 and HIS49/CD59 assays, as screening tools for in vivo mutagenicity. We used both the CD45/CD59 and/ or HIS49/CD59 protocols to monitor the kinetics of Pig$a$ mutant frequency, as measured by the frequency of CD59-deficient RBCs in rats treated with standard genotoxic chemicals (ENU, cyclophosphamide (CP), 4-nitroquinoline-1-oxide (4-NQO), and ethylmethanesulfonate (EMS)). We also compared the kinetics of Pig-a mutant manifestation to that of micronucleus induction in rats treated with ENU or CP. Finally, we evaluated the comparability and relative sensitivity of the Pig- $a$ mutant response produced in CD45-negative cells and HIS49positive RBCs. 
Rat Pig-a mutation assay for mutagenicity screening

\section{MATERIALS AND METHODS}

\section{Reagents}

ENU, EMS, and ethylenediaminetetraacetic acid (EDTA) were purchased from Sigma-Aldrich (St. Louis, MO, USA). 4-NQO and methylcellulose (50 cps) were purchased from Wako-Pure Chemical Industry Co., Ltd. (Tokyo, Japan). Cyclophosphamide hydrate (Endoxan ${ }^{\circledR}$; CP) was purchased from Shionogi \& Co., Ltd. (Osaka, Japan). Calcium- and magnesium-free phosphatebuffered saline (PBS, pH 7.2) was purchased from Invitrogen, (Carlsbad, CA, USA), while saline (Otsuka Normal Saline) was purchased from Otsuka Pharmaceutical Factory, Inc. (Tokushima, Japan).

Anti-rat CD59 antibody (clone TH9, FITC-conjugated), anti-rat CD45 antibody (clone OX-1, PECy5-conjugated), anti-rat erythroid marker (clone HIS49, biotinconjugated), and streptavidin-APC were purchased from BD Biosciences (Tokyo, Japan). In vivo Rat MicroFlow ${ }^{\circledR}$ Kits, for flow cytometric micronucleus scoring, were purchased from Litron Laboratories (Rochester, NY, USA).

\section{Animals, administration of chemicals and blood collection}

All experimental procedures were conducted in accordance with the Guiding Principles for the Care and Use of Laboratory Animals (Teijin Pharma Ltd., Tokyo, Japan), and each experimental protocol was approved by the Committee for Animal Experiments of the Teijin Institute for Biomedical Research. Male $\mathrm{CD}(\mathrm{SD})$ rats were obtained from Charles River Laboratories (Yokohama, Japan); the rats received feed and water ad libitum. Rats were administrated test agents by gavage after a 7 to 12-day acclimation period.

A summary of the experiments conducted for this study is shown in Table 1. ENU was dissolved in warm $\left(37^{\circ} \mathrm{C}\right)$ PBS ( $\mathrm{pH}$ adjusted to 6.0-6.1) at a concentration of $10 \mathrm{mg} / \mathrm{ml}$ (ENU concentration adjusted to account for the water and acetic acid stabilizer in the commercial preparation), sterilized with a $0.2 \mu \mathrm{m}$ filter, and diluted to 1.0 and $4.0 \mathrm{mg} / \mathrm{ml}$. Rats were treated with four weekly doses of 0,10 , or 40 $\mathrm{mg} / \mathrm{kg}$ ENU, or with single doses of 0 or $40 \mathrm{mg} / \mathrm{kg}$. CP was dissolved in PBS ( $\mathrm{pH} 7.2)$ at a concentration of 10 $\mathrm{mg} / \mathrm{ml}$ and diluted to 0.5 and $2.0 \mathrm{mg} / \mathrm{ml}$. Rats were treated with 10 daily doses of 0 or $5 \mathrm{mg} / \mathrm{kg} \mathrm{CP}$ except for the weekend, or single dose of $20 \mathrm{mg} / \mathrm{kg} \mathrm{CP.} \mathrm{4-NQO} \mathrm{was} \mathrm{dis-}$ solved in $0.5 \%(\mathrm{w} / \mathrm{v})$ methylcellulose in distilled water and diluted to $5.0,10$, and $20 \mathrm{mg} / \mathrm{ml}$. Rats were treated with single doses of $0,50,100$, or $200 \mathrm{mg} / \mathrm{kg} \mathrm{4-NQO.}$ EMS was dissolved in PBS (pH 7.2) and saline, in Experiment 1 and Experiment 2, respectively. EMS used in
Experiment 1 was diluted to 9.0 and $36 \mathrm{mg} / \mathrm{ml}$. Rats were treated with four weekly doses of 0 or $90 \mathrm{mg} / \mathrm{kg}$ or with a single dose of $360 \mathrm{mg} / \mathrm{kg}$ EMS, respectively. In Experiment 2, EMS was diluted to 1.2, 2.0, 18, and $36 \mathrm{mg} / \mathrm{ml}$. Rats were treated with 28 daily doses of 0,12 , or $20 \mathrm{mg} / \mathrm{kg}$ EMS, or with single doses of 180 or $360 \mathrm{mg} / \mathrm{kg}$ EMS, respectively. All preparations were administrated to rats in a volume of $10 \mathrm{ml} / \mathrm{kg}$.

Blood was collected from the tail vain of treated animals as described in Table 1. All PB was promptly mixed with an EDTA solution ( 9 parts blood to 1 part $12 \mathrm{mg} / \mathrm{ml}$ EDTA).

\section{Pig-a assay}

Two distinct staining strategies (CD45/CD59 method and HIS49/CD59 method) were conducted for this study. For the CD45/CD59 method, $1 \mu$ of blood/EDTA mixture was suspended in $0.2 \mathrm{ml}$ of PBS, and the cells were labeled with $1 \mu \mathrm{g}$ of PECy5-conjugated anti-rat CD45 antibody and $1 \mu \mathrm{g}$ of FITC-conjugated anti-rat CD59 antibody. After incubation for $1 \mathrm{hr}$ in the dark at room temperature, the cells were washed with PBS, centrifuged $5 \mathrm{~min}$ at $1,680 \times \mathrm{g}$, and resuspended in $1 \mathrm{ml}$ of PBS. Flow cytometry (FCM) analysis of both CD45 and CD59-negative cells was conducted on a EPICS-XL flow cytometer equipped with a $488 \mathrm{~nm}$ blue laser (Beckman Coulter, Tokyo, Japan). Typical cytograms are shown in Fig. 1A. A single cell population was gated by light scatter, and then CD45-positive white blood cells were excluded from this population by gating on CD45-negative cells. Approximately 300,000 to 500,000 CD45-negative cells (presumed RBCs) were analyzed further for expression of CD59 on the cell surface. For the HIS49/CD59 method, $3 \mu \mathrm{l}$ of blood/EDTA mixture were suspended in $200 \mu \mathrm{l}$ of PBS, and the cells were labeled with $0.25 \mu \mathrm{g}$ of biotinylated HIS49 antibody and $1 \mu \mathrm{g}$ of FITC-conjugated anti-rat CD59. After incubation for $1 \mathrm{hr}$ in the dark at room temperature, the samples were washed with PBS, centrifuged $5 \mathrm{~min}$ at 1,680 $\times \mathrm{g}$, and resuspended in $200 \mu \mathrm{l}$ of PBS; $0.25 \mu \mathrm{g}$ of APC-conjugated streptavidin were added, and the samples were incubated for $15 \mathrm{~min}$ in the dark at room temperature. The cell samples then were centrifuged $5 \mathrm{~min}$ at $1,680 \times g$ and resuspended in $1 \mathrm{ml}$ of PBS. FCM analysis of HIS49-positive and CD59negative RBCs was conducted on a FACSAria ${ }^{\mathrm{TM}}$ I flow cytometer equipped with $405 \mathrm{~nm}$ violet, $488 \mathrm{~nm}$ blue, and $635 \mathrm{~nm}$ red lasers (BD Biosciences, Tokyo, Japan). Typical cytograms are shown in Fig. 1B. A single cell population was gated by light scatter, and then HIS49-positive RBCs were gated. Approximately 300,000 to 500,000 HIS49-positive RBCs were analyzed further for expres- 
T. Kimoto et al.

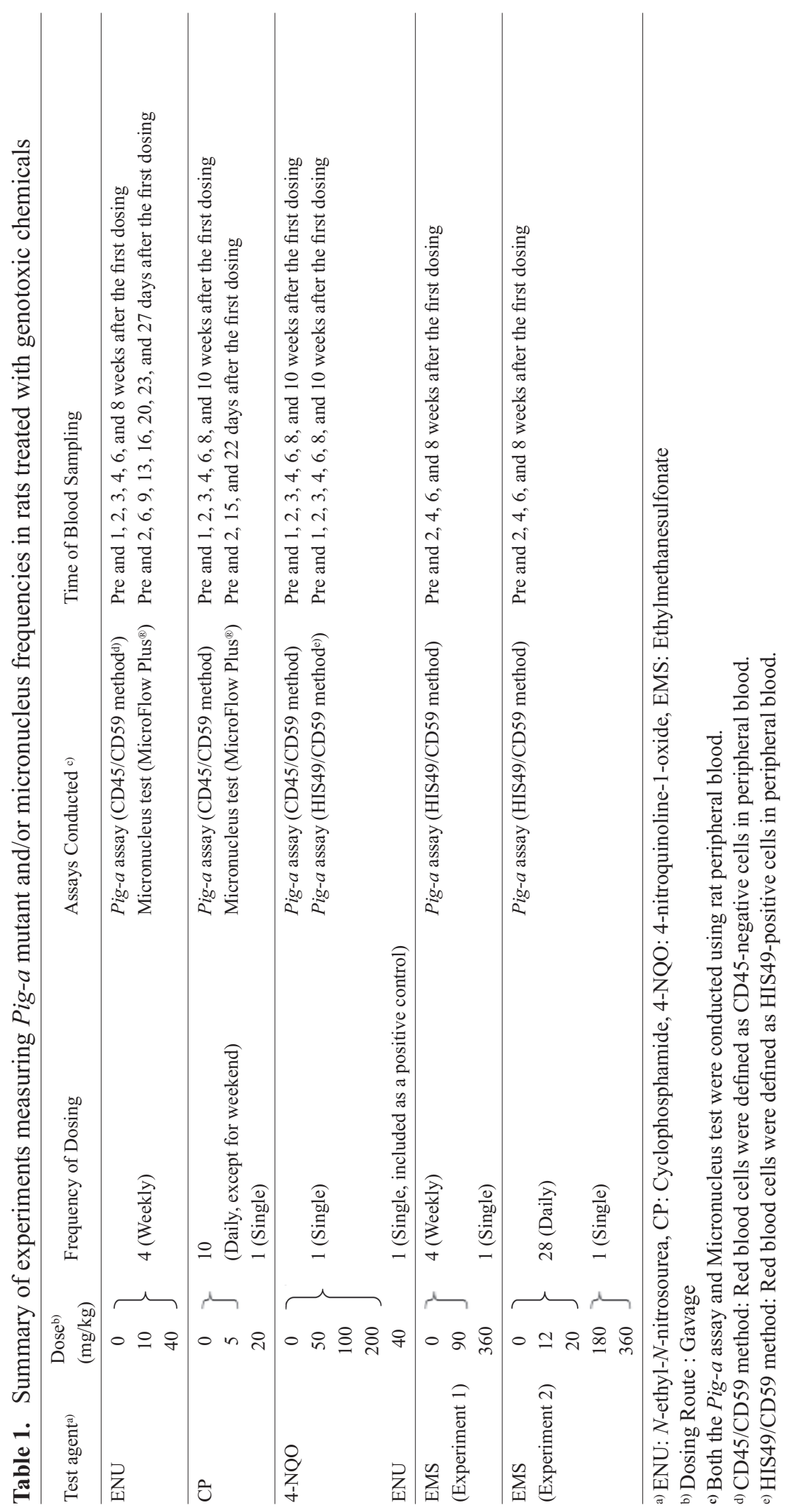

Vol. 37 No. 5 


\section{(A)}
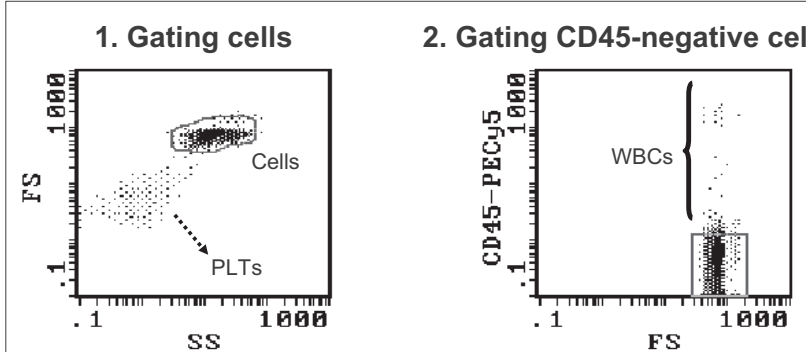

3. CD59 expression analysis

(B)

\section{Gating cells}

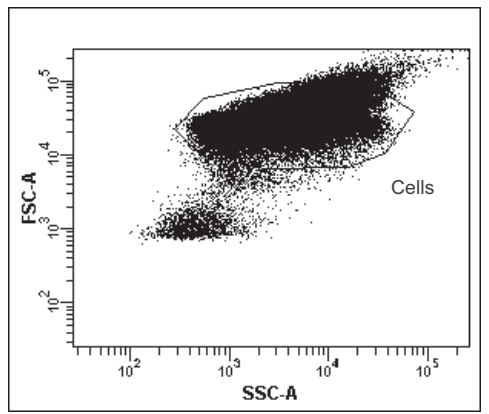

2. Gating HIS49-positive cells

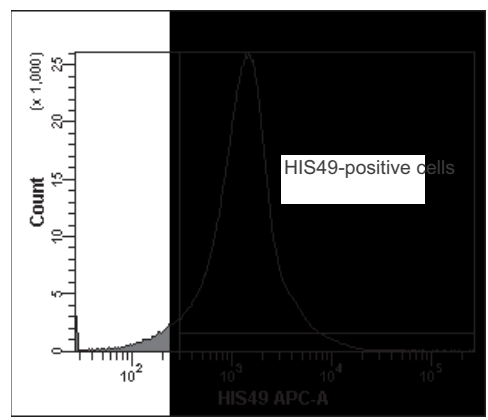

3. CD59 Expression Analysis

Control (PBS)

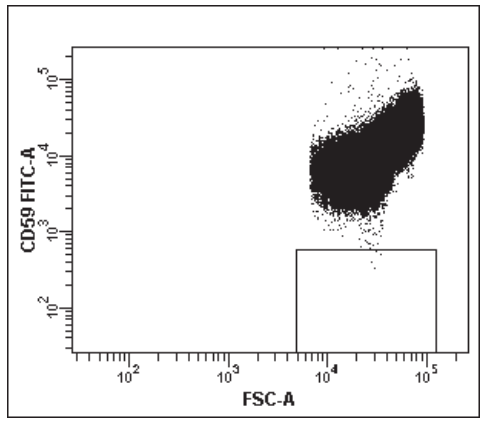

$40 \mathrm{mg} / \mathrm{kg}$ ENU

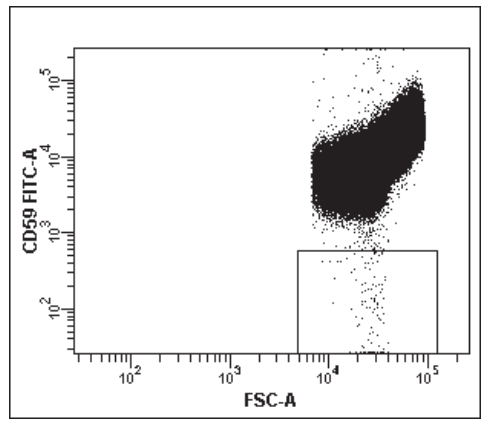

Fig. 1. Flow cytometry analysis for detection of CD59-negative RBCs. (A) For analysis with the CD45/CD59 protocol, cells first were gated by light scatter to exclude the platelets and then for the expression of CD45 (pan-leukocyte marker). CD45-negative cells (the presumed RBC population) were gated and analyzed for CD59 expression. (B) For analysis with the HIS49/ CD59 protocol, cells first were gated by light scatter and then for the expression of rat erythroid marker, HIS49. HIS49positive cells were gated and analyzed for CD59 expression.

sion of CD59 on the cell surface.

No fluorescence compensation was employed for either method. All gates for detecting CD59-negative mutant RBCs were set using unstained and single-antibodystained samples before evaluating the multi-antibody- labeled experimental samples. As described by Kimoto et al. (2011a), the frequency of CD59-negative RBCs in antibody-stained blood samples was estimated from the number of CD59-negative RBCs falling into the mutant gate and the total number of RBCs interrogated. 


\section{Micronucleus assay}

Micronucleus assays using rat PB samples were conducted with In vivo Rat MicroFlow ${ }^{\circledR}$ Kits. All samples were prepared according to the instruction manual in the kit. Approximately 20,000 CD71-positive RETs were interrogated for the frequency of miconuclei-positive RETs (MN-RET\%) and CD71-positive RETs (\%) using a FACSAria $^{\mathrm{TM}}$ I flow cytometer (BD Biosciences).

\section{Statistical analysis}

Dunnett's multiple comparison t-test or Student's t-test was used for the statistical analysis. For the ENU, 4-NQO and EMS (Experiment 2) study, Dunnett's multiple comparison t-test was used for comparisons between the vehicle control group and each of the treatment groups at each time point. For the CP and EMS (Experiment 1) study, Student's t-test was used for comparisons between the control group and each treatment group at each time point because there were two treatment groups with different numbers of doses. Levels of significance were expressed as $\mathrm{p}<0.05, \mathrm{p}<0.01$, or $\mathrm{p}<0.001$.

\section{RESULTS}

\section{ENU: Monitoring Pig-a mutant kinetics and micronucleus induction}

In the ENU experiment, rats were administered 0, 10, or $40 \mathrm{mg} / \mathrm{kg}$ ENU in four weekly doses, and Pig-a mutant frequency was measured as the frequency of $\mathrm{CD}_{4} 5^{-}$ CD59- RBCs before dosing (Pre), and at 1, 2, 3, 4, 6, and 8 weeks after the first dosing. In addition, MN-RET\% and the percentage of CD71-positive RETs among total erythrocytes were scored at Pre, 2, 6, 9, 13, 16, 20, 23, and 27 days after the first dosing. Transient decreases in CD71positive RETs (\%) were found 2 days after each dose of 10 and $40 \mathrm{mg} / \mathrm{kg}$ ENU, compared to that of vehicle control (Fig. 2A). Dose related increases in Pig-a mutant frequency were found in the ENU treatment groups, and these mutant frequencies increased steadily over the 8 week observation period (Fig. 2B). Micronucleus induction in the same ENU treated rats was increased at 2 days after the each treatment, but then diminished to near control levels within 1 week after the treatments. Thus, the kinetics of the response and the persistence of the response were different for Pig-a mutant frequency and micronucleus induction (Fig. 2B).

\section{CP: Monitoring Pig-a mutant kinetics and micronucleus induction}

In the $\mathrm{CP}$ experiment, rats were administered either
(A)

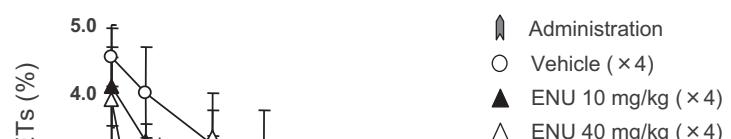

$\triangle \mathrm{ENU} 40 \mathrm{mg} / \mathrm{kg}(\times 4)$

(B)

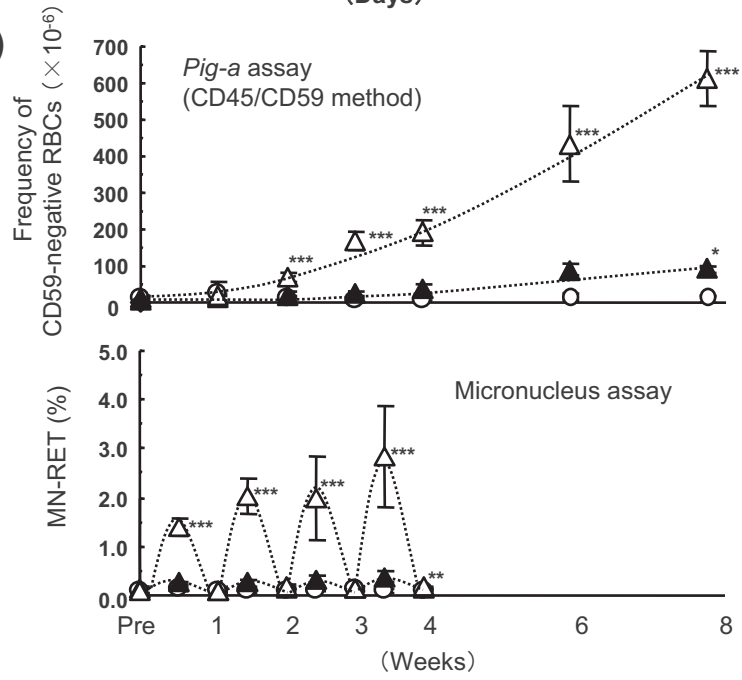

Fig. 2. Percent of CD71-positive RETs among total RBCs (A) and the kinetics of micronucleated RET frequency (MN-RET\%) and the frequency of CD59-negative RBCs (B) in rats treated with $N$-ethyl- $N$-nitrosourea (ENU). All data are the mean \pm S.D. of data from five animals in each group. Dark arrows indicate the time of treatment. Doses of ENU were as follows: $0 \mathrm{mg} / \mathrm{kg}$ $\times 4$ weekly doses (open circles), $10 \mathrm{mg} / \mathrm{kg} \times 4$ weekly doses (solid triangles), and $40 \mathrm{mg} / \mathrm{kg} \times 4$ weekly doses (open triangles). Statistically significant differences from vehicle controls are indicated at the $\mathrm{p}<0.05$, 0.01 , or 0.001 levels $(*, * *$, or $* * *$, respectively).

10 daily doses of 0 or $5 \mathrm{mg} / \mathrm{kg} \mathrm{CP}$ except for the weekend, or a single dose of $20 \mathrm{mg} / \mathrm{kg} \mathrm{CP}$. Pig- $a$ mutant frequency was measured as the frequency of CD $45^{-} / \mathrm{CD} 59^{-} \mathrm{RBCs}$ at Pre, 1, 2, 3, 4, 6, 8, and 10 weeks after the first dosing. In addition, MN-RET\% and the percentage of CD71-positive RETs among total erythrocytes were scored at Pre and 2, 15, and 22 days after the first dosing. A transient decrease in CD71-positive RETs (\%) was found 2 days after the single dose of $20 \mathrm{mg} / \mathrm{kg} \mathrm{CP}$, compared to that of the vehicle control (Fig. 3A). Also, a persistent decrease in CD71- 
Rat Pig-a mutation assay for mutagenicity screening

positive RETs (\%) was found as a result of the repeated doses of $5 \mathrm{mg} / \mathrm{kg} \mathrm{CP}$. These decreases recovered to vehicle control levels at 1 or 2 weeks after the final dosing. Except at 4 weeks after the first treatment with $5 \mathrm{mg} / \mathrm{kg}$ CP, no significant increases in Pig-a mutant frequency were found at any of the sampling points (Fig. 3B). Micronucleus induction in the same CP-treated rats was increased at 2 days after the first treatment of $20 \mathrm{mg} / \mathrm{kg}$ $\mathrm{CP}$ and at 2 and 15 days after the first treatment of $5 \mathrm{mg} / \mathrm{kg}$ $\mathrm{CP}$, respectively. These increases decreased to the levels in vehicle control rats at 1 or 2 weeks after the final dosing; thus micronucleus induction was a transient response (Fig. 3B).

\section{4-NQO: Monitoring Pig-a mutant kinetics with two distinct approaches}

Figure 4 shows the time course for Pig-a mutant frequency manifestation in rats administered single doses of $0,50,100$, or $200 \mathrm{mg} / \mathrm{kg}$ 4-NQO. Pig- $a$ mutant frequency was measured as both the frequency of $\mathrm{CD} 45^{-/ \mathrm{CD} 59^{-}}$ RBCs and the frequency of HIS $49^{+} / \mathrm{CD}^{-} 9^{-} \mathrm{RBCs}$ at Pre, $1,2,3,4,6,8$, and 10 weeks. Because of severe toxicity, the animals in the $200 \mathrm{mg} / \mathrm{kg}$ treatment group were euthanized 1 week after the treatment. Compared with the spontaneous Pig- $a$ mutant frequencies, clear time- and doserelated increases in mutant frequency were detected in the treated rats, until a plateau was reached approx. 6 weeks after the 4-NQO treatments. The elevated mutant frequencies persisted for the remainder of the 10 week observation period. The kinetics of mutant manifestation and the induced mutant frequencies were similar using both staining protocols (Fig. 4A, 4B).

\section{EMS: Monitoring of Pig-a mutant kinetics in HIS49-positive RBCs}

We performed two experiments using the HIS49/CD59 method to monitor Pig-a mutant kinetics in rats treated with EMS. Figure 5 shows the results of Experiment 1 in which rats were treated with four weekly doses of 0 or $90 \mathrm{mg} / \mathrm{kg}$ EMS or a single dose of $360 \mathrm{mg} / \mathrm{kg}$ EMS. Pig- $a$ mutant frequency was measured as the frequency of HIS49 ${ }^{+} \mathrm{CD}^{-} 9^{-}$RBCs at Pre, 2, 4, 6, and 8 weeks after the first dosing. Mean spontaneous Pig-a mutant frequencies in vehicle control rats ranged from $3.9 \times 10^{-6}$ to $7.4 \times 10^{-6}$ (Fig. 5). The maximum response with the single dose of $360 \mathrm{mg} / \mathrm{kg}$ was $17.8 \times 10^{-6}$ at 4 weeks after dosing, which was a significant increase in mutant frequency. As for the four weekly doses of $90 \mathrm{mg} / \mathrm{kg}$ (total $360 \mathrm{mg} / \mathrm{kg}$ ), the maximum response was $13.9 \times 10^{-6}$ at 6 weeks after the first dosing. While this response was not significantly increased relative to the control, the increases at the other
(A)

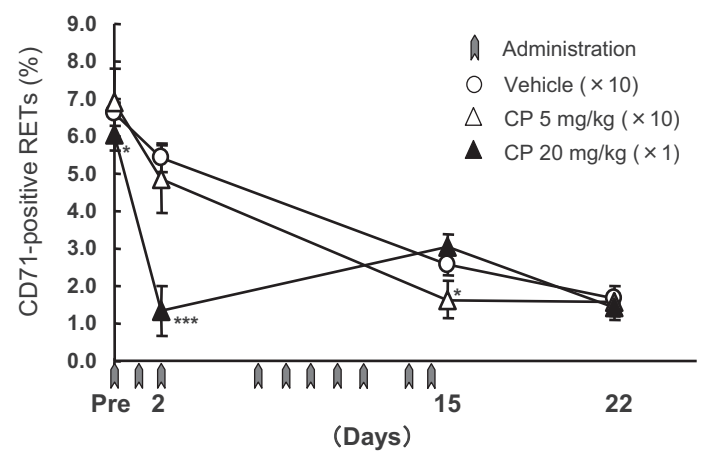

(B)

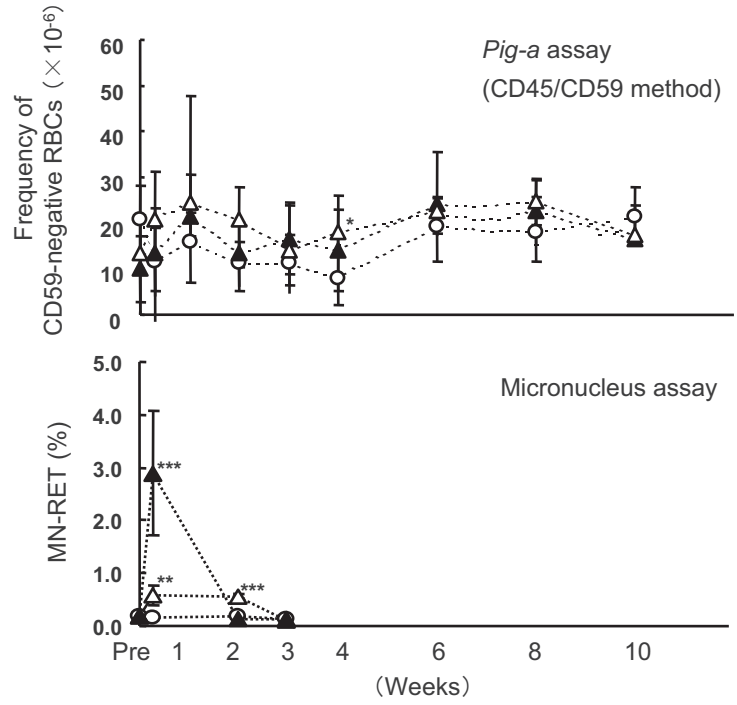

Fig. 3. Percent of CD71-positive RETs among total RBCs (A) and the kinetics of MN-RET\% and the frequency of CD59-negative RBCs (B) in rats treated with cyclophosphamide (CP). All data are the mean \pm S.D. of data from five animals in each group. Dark arrows indicate the time of treatment. Doses of CP were as follows: $0 \mathrm{mg} / \mathrm{kg} \times 10$ daily doses (open circles), $5 \mathrm{mg} / \mathrm{kg} \times 10$ daily doses (open triangles), and single dose of $20 \mathrm{mg} / \mathrm{kg}$ (solid triangles). Statistically significant differences from vehicle controls are indicated at the $p<0.05$, 0.01 , or 0.001 levels $(*, * *$, or $* * *$, respectively).

sampling times were significant.

In Experiment 2, rats were dosed orally for 28 days with vehicle or with 12 or $20 \mathrm{mg} / \mathrm{kg}$ EMS, or with single treatments of 180 or $360 \mathrm{mg} / \mathrm{kg}$ EMS. Pig- $a$ mutant frequency was measured as the frequency of HIS49+/CD59RBCs at Pre, 2, 4, 6, and 8 weeks after the first dosing. Mean spontaneous Pig- $a$ mutant frequencies in pre-dosing rats ranged from $2.2 \times 10^{-6}$ to $6.7 \times 10^{-6}$ (Data not shown). All the individual Pig- $a$ mutant frequencies are exhibited on scatter plots (Fig. 6). Mean spontaneous Pig- 
(A)

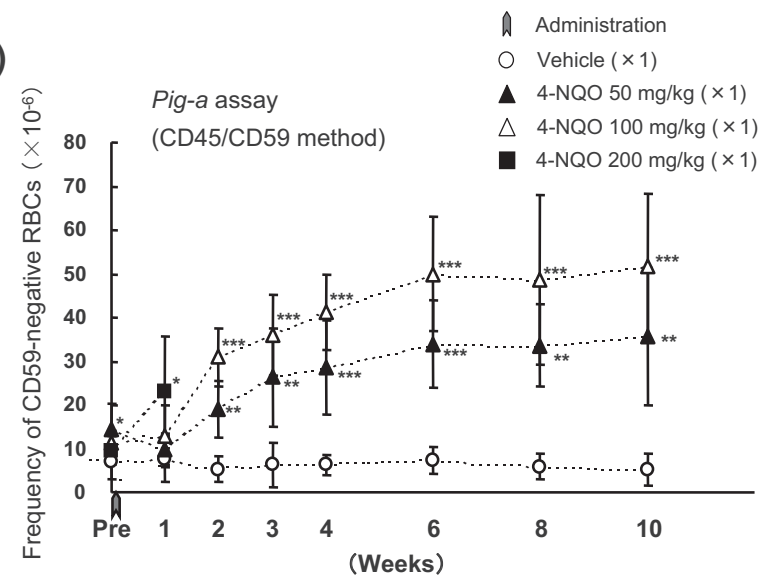

(B)

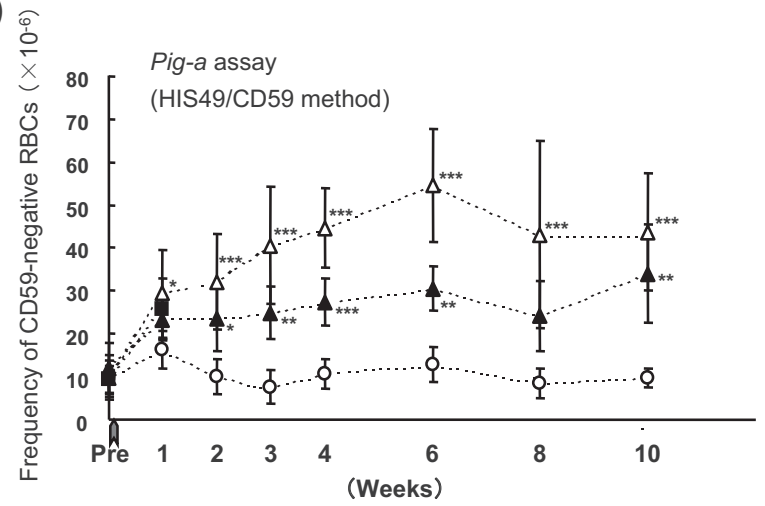

Fig. 4. Frequencies of CD59-negative RBCs in rats treated with 4-nitroquinoline-1-oxide (4-NQO). Dark arrow indicates the time of treatment. Doses of 4-NQO were as follows: $0 \mathrm{mg} / \mathrm{kg}$ (open circles), $50 \mathrm{mg} / \mathrm{kg}$ (solid triangles), $100 \mathrm{mg} / \mathrm{kg}$ (open triangles), and $200 \mathrm{mg} / \mathrm{kg}$ (solid squares). (A): The frequency of CD59-negative RBCs using the CD45/CD59 protocol. (B): The frequency of CD59-negative RBCs using the HIS49/ CD59 protocol. All data are the mean \pm S.D. of data from six animals in each group, except the $200 \mathrm{mg} / \mathrm{kg}$ group was $n=4$. Statistically significant differences from vehicle controls are indicated at the $\mathrm{p}<0.05$, 0.01 , or 0.001 levels $(*, * *$, or $* * *$, respectively).

$a$ mutant frequencies in the vehicle control group at 2, 4 , 6 , and 8 weeks after the first dosing were $2.2 \times 10^{-6}, 3.3 \times$ $10^{-6}, 0.0 \times 10^{-6}$, and $3.0 \times 10^{-6}$, respectively (Fig. 6A, 6B, $6 \mathrm{C}$, and $6 \mathrm{D}$ ). Consistent with the findings of Experiment 1, a single dose of $360 \mathrm{mg} / \mathrm{kg}$ EMS increased the mean Pig- $a$ mutant frequencies to more than $10 \times 10^{-6}$ at 4 and 6 weeks after dosing; a similar response was found for the repeat dose of $20 \mathrm{mg} / \mathrm{kg}$ EMS at 6 and 8 weeks after the first dosing.

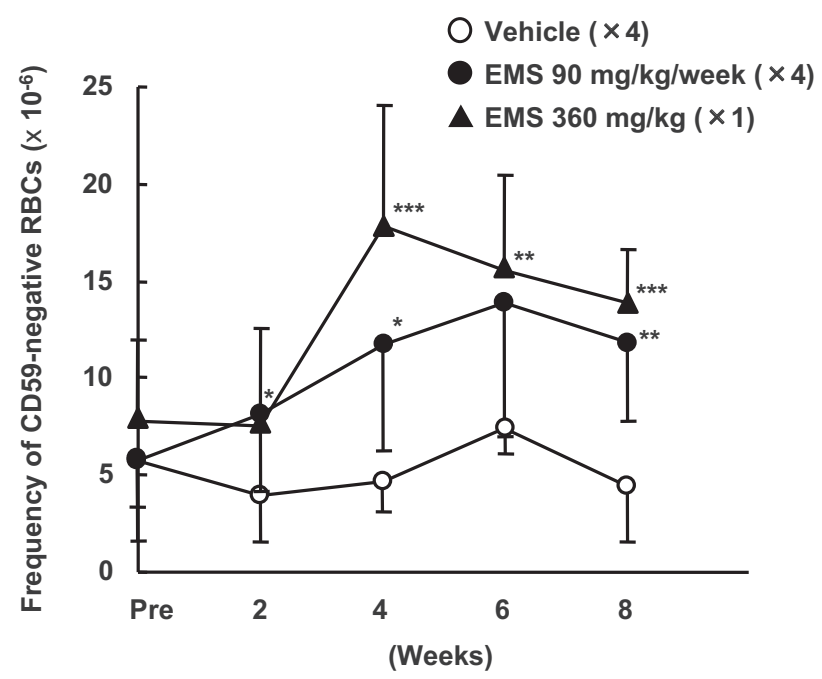

Fig. 5. Frequencies of CD59-negative RBCs in rats treated with ethylmethanesulfonate (EMS) using the HIS49/ CD59 method. Doses of EMS were as follows: $0 \mathrm{mg} / \mathrm{kg}$ $\times 4$ weekly doses (open circles), $90 \mathrm{mg} / \mathrm{kg} \times 4$ weekly doses (solid triangles), and single dose of $360 \mathrm{mg} / \mathrm{kg}$ (solid circles). All data are the mean \pm S.D. of data from six animals in each group. Statistically significant differences from vehicle controls are indicated at the $\mathrm{p}<0.05,0.01$, or 0.001 levels $\left(^{*}, * *\right.$, or $* * *$, respectively).

\section{Comparability and sensitivity of the Pig-a mutant response using the two assay methods}

In order to confirm the comparability of the Pig-a mutant responses as measured by the CD45/CD59 method and the HIS49/CD59 method, we compared the frequency of CD59-negative RBCs from individual rats following single treatment of $40 \mathrm{mg} / \mathrm{kg} \mathrm{ENU}$, and measured using both methods. Frequencies of CD59-negative RBCs increased similarly with time and dose using both methods. A positive correlation was observed between the frequencies of CD45-/CD59- RBCs and the frequencies of HIS49 ${ }^{+} / \mathrm{CD} 59-\mathrm{RBCs}$ within the same animals $\left(r^{2}=0.8\right.$, Fig. 7). These data were collected simultaneously in the experiments conducted with 4-NQO as a positive control group ( $n=4 /$ group), so that the blood-sampling schedule for the ENU-treated rats is the same as the schedule described in Table 1 for 4-NQO-treated rats.

We also compared our in-house historical background/ vehicle control data (April 2008 to December 2011) for the frequencies of Pig- $a$ mutation measured either as CD45-/CD59- RBCs or HIS49+/CD59- RBCs (Fig. 8). The mean background frequency using the CD45/CD59 method was $12.7 \times 10^{-6} .17 .7 \%(48 / 271)$ of the mutant frequen- 
Rat Pig- $a$ mutation assay for mutagenicity screening

(A)

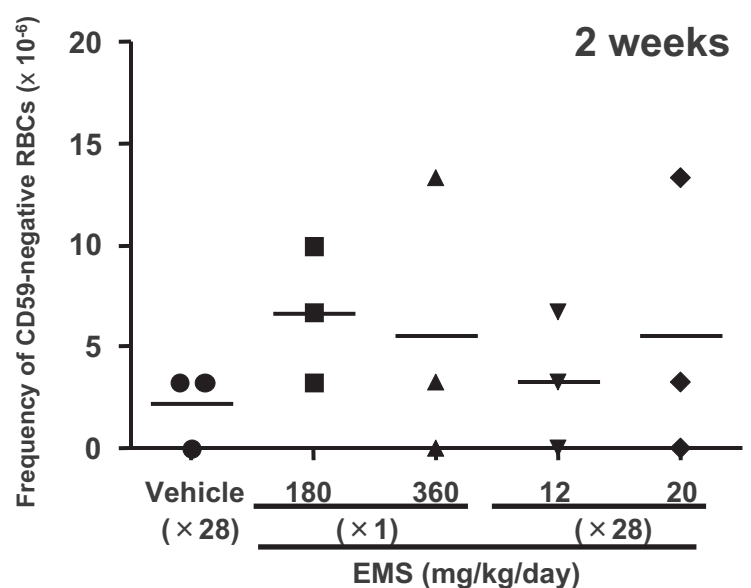

(B)

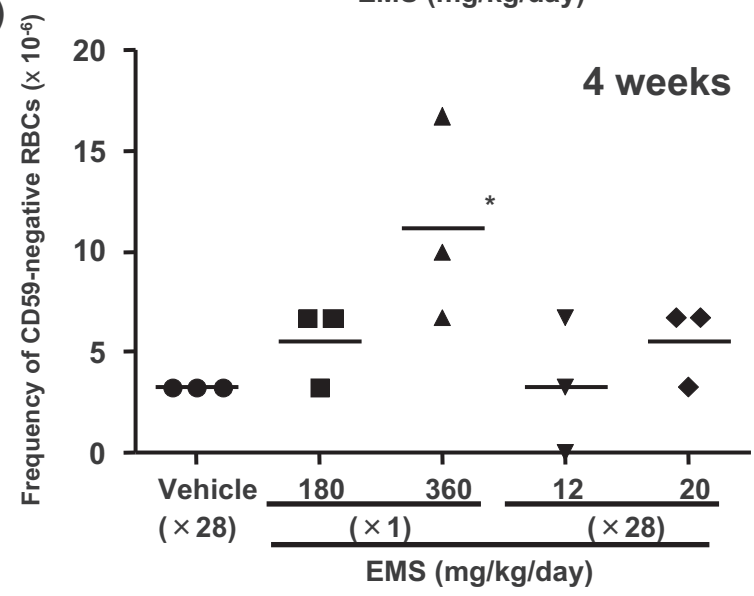

(C)

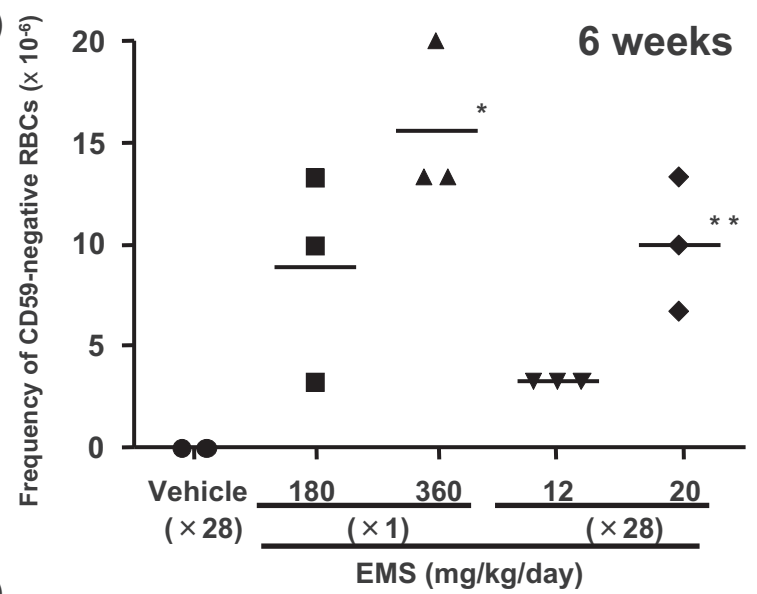

(D)

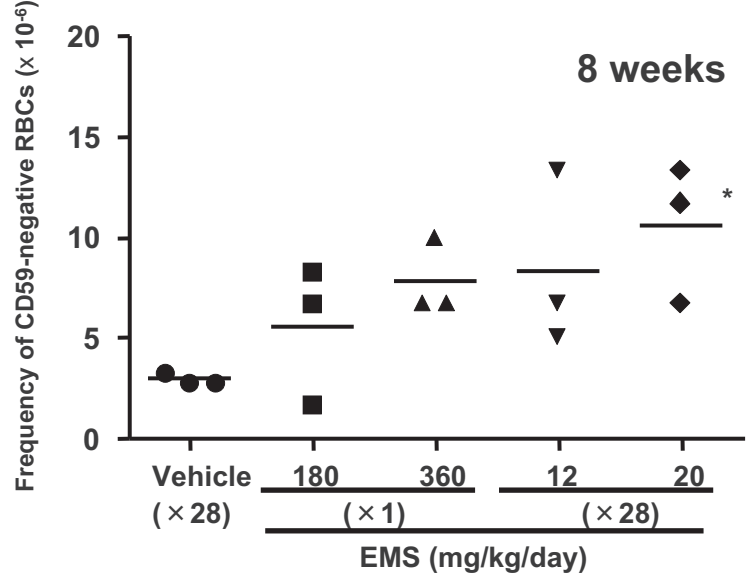

Fig. 6. Frequencies of CD59-negative RBCs in rats treated with a single dose (180 or $360 \mathrm{mg} / \mathrm{kg})$ or 28 days of repeat dosing (12 or $20 \mathrm{mg} / \mathrm{kg}$ ) with EMS. Mutant frequencies were analyzed using the HIS49/CD59 method. Figure 6(A), (B), (C), and (D) are the data from assays conducted at 2, 4, 6, and 8 weeks after the first dosing, respectively. All individual data are exhibited as scatter plots and a bar shows the mean of each group. Statistically significant differences from vehicle controls are indicated at the $\mathrm{p}<0.05$, or 0.01 levels $(*$, or $* *$, respectively).

cies were less than $5 \times 10^{-6}$, and $56.1 \%(152 / 271)$ less than $10 \times 10^{-6}$. As for the HIS49/CD59 method, the mean background frequency was $5.5 \times 10^{-6} .60 .1 \%(104 / 173)$ of the mutant frequencies were less than $5 \times 10^{-6}$, and $86.7 \%$ $(150 / 173)$ less than $10 \times 10^{-6}$.

\section{DISCUSSION}

The objective of this study was to compare two variations of an anti-CD59-based Pig-a assay (the CD45/ CD59 and HIS49/CD59 methods) and evaluate their potential as screening tools for in vivo mutation by monitoring the kinetics of Pig-a mutant frequency in rats treated with well-known genotoxic chemicals. In addition, the Pig- $a$ mutant frequency response was compared to the response of the most commonly used in vivo genotoxicity assay, the micronucleus assay. Then the comparability and assay sensitivity of Pig-a mutant frequency detected by the CD45/CD59 and HIS49/CD59 methods were compared.

The potent gene mutagen, ENU, was shown in several earlier studies to produce large increases in Pig$a$ mutant frequency in dosed rats (e.g., Cammerer et al., 2011; Dertinger et al., 2010; Miura et al., 2009). In this study, we compared the kinetics of Pig-a mutant frequency and micronucleus induction in rats after four weekly doses of ENU. While Pig- $a$ mutant frequency accumulated and persisted in a time- and dose-related manner for at least 8 weeks after the first treatment, micronucleus induction was maximum at 2 days and diminished to 
T. Kimoto et al.

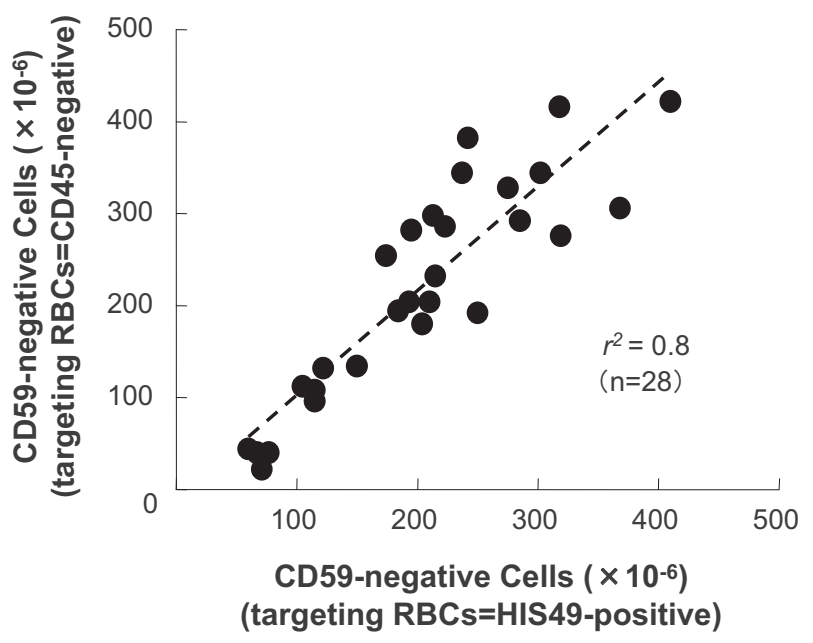

Fig. 7. Comparison of two distinct Pig- $a$ assay protocols for measuring the frequency of CD59-negative RBCs. Data were from individual rats at $1,2,3,4,6,8$, and 10 weeks following single treatments of $40 \mathrm{mg} / \mathrm{kg}$ ENU.

near control levels within 1 week after the each treatment. These results demonstrate that the manifestation kinetics and persistence of these two endpoints are very different. Previous Pig- $a$ mutant manifestation studies indicate that ENU-induced Pig- $a$ mutant RBCs in rats persist for at least 6 months after the first treatment with ENU, and that Pig- $a$ mutant RBCs accumulate after repeat dosing in a near-additive fashion (Dertinger et al., 2010; Phonethepswath et al., 2010; Miura et al., 2009). Micronucleus induction is usually short-lived after single doses, but pseudo-persistent responses can appear to occur after daily repeated doses (Hamada et al., 2001a). Thus, it would be possible to evaluate both chromosomal aberration and gene mutation if the Pig- $a$ assay and the micronucleus assay were integrated into 28-day toxicology studies. Recent reports support this possibility (Cammerer et al., 2011; Dertinger et al., 2010).

$\mathrm{CP}$ is an alkylating agent that is often used as a positive control for the micronucleus test (Hamada et al., 2001b). Similar to the results of the ENU experiment, a transient increase in MN-RET\% was found after each treatment with CP. However, the Pig- $a$ mutant frequency response following CP treatment was extremely weak, and, with the exception of a sample evaluated 4 weeks after the first treatment with $5 \mathrm{mg} / \mathrm{kg} \mathrm{CP}$, no statistically significant increases in Pig-a mutant frequency were found in this present study. In addition, the one positive measurement occurred at the point when the Pig- $a$ mutant
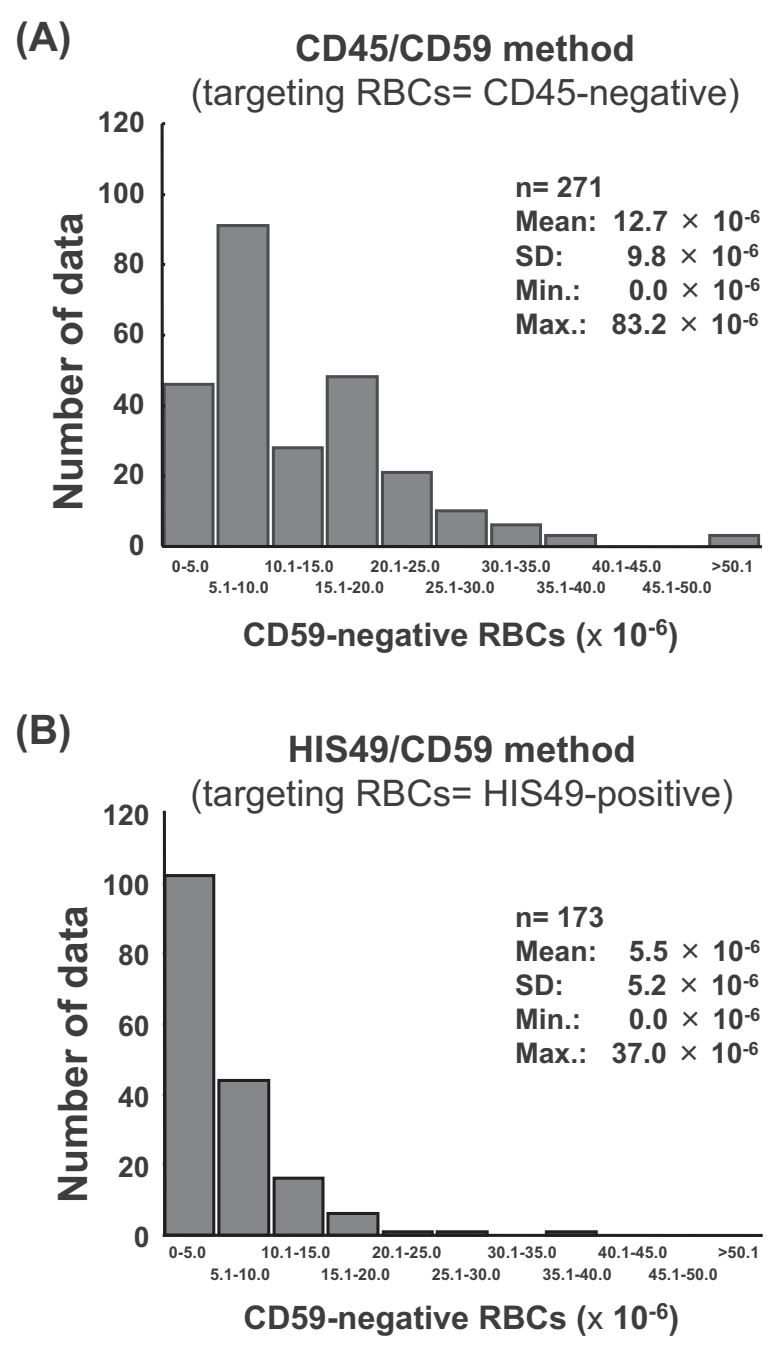

Fig. 8. Distributions of background Pig-a mutant frequencies measured using two distinct Pig- $a$ assay methods. Pig$a$ mutant frequency was measured as the frequency of CD59-deficient RBCs. (A); background Pig- $a$ mutant frequencies measured with the CD45/CD59 protocol. (B); background Pig-a mutant frequencies measured with the HIS49/CD59 protocol. All data were from individual rats treated with vehicles as part of assays conducted at Teijin Pharma from April 2008 to December 2011.

frequency in vehicle control was at its lowest in the study. Thus, the biological significance of this increase in CPtreated rats is questionable, Walker et al. (2004) reported that the Hprt mutant lymphocyte frequency 8 weeks after treating rats with $20 \mathrm{mg} / \mathrm{kg} \mathrm{CP}$ was approx. 2- or 3 -times higher than the frequency in control rats. These results suggest that the mutagenic potential of $\mathrm{CP}$ is not 
Rat Pig-a mutation assay for mutagenicity screening

strong, at least for genes on the X-chromosome like Pig$a$ or Hprt, and that a spontaneous Pig-a mutant frequency as low and as stable as practical may be necessary for detecting any mutagenicity induced by $\mathrm{CP}$.

4-NQO forms stable quinoline monoadducts with purine bases through the enzymatic reduction of its nitro group (Kondo, 1977). A previous report described timeand dose-dependent increases of Pig-a mutant RBCs in rats treated with 4-NQO (Stankowski et al., 2011). In the present study, we demonstrated that both the CD45/CD59 and HIS49/CD59 assay detected similar Pig- $a$ mutant responses. The major difference between the assays is that the CD45/CD59 assay assumes CD45-negative events are erythrocytes, whereas the HIS49/CD59 assay more directly identifies erythroid cells using a fluorescent antibody. These 4-NQO experiments and the comparison of data shown in Fig. 7 support the conclusion that these protocols are comparable, at least in terms of detecting induced mutant responses.

Significant increases in Pig-a mutant frequency were found after single and 4 weekly doses of EMS. Although the accumulation and the persistence of Pig-a mutant frequency were very similar for both treatment schedules during the 8 week observation period, the maximum responses in the single dose group and the 4 weekly dose group were found at 4 weeks and 6 weeks, respectively, although in the latter case the increase was not significant. This difference was consistent with a time lag in the rate of increase due to the fractionated dosing. A single dose of $360 \mathrm{mg} / \mathrm{kg}$ EMS produced similar small increases in Pig- $a$ mutant frequency in both Experiments 1 and 2. In addition, small increases in Pig- $a$ mutant frequency were detected in the 28-day repeat dosing with $20 \mathrm{mg} / \mathrm{kg}$ EMS at 6 and 8 weeks after the first dosing. Because only three animals were used in our dosing groups, our data may have suffered from type II error and therefore should be treated with caution. However, Dobo et al. (2011), using another approach to measure Pig-a mutant frequency, also found that measurement of Pig-a mutant RBCs was a useful method for measuring mutation in rats treated with EMS. Recent studies show that EMS exhibits a threshold for induced genotoxicity (Gocke et al., 2009; Muller et al., 2009) and it has been observed that, given the relative ease with which mutant frequency data can be generated with the Pig-a assay, the assay has potential for examining the possibility of in vivo genotoxicity thresholds for other DNA-reactive compounds (Dobo et al., 2011). Our results suggest that the methods we have developed to measure Pig- $a$ mutant RBCs might be useful for developing detailed in vivo dose responses for genotoxic agents; however, more work is needed to estab- lish the accuracy and reliability of data generated by these Pig- $a$ assays.

There was a positive correlation between the frequencies of CD45/CD59 RBCs and the frequencies of HIS49/ CD59 RBCs within same animals following single treatments with $40 \mathrm{mg} / \mathrm{kg}$ ENU (Fig. 7). The data suggest that both methods measure comparable frequencies of Pig-a mutant RBCs. Our in-house historical background data, however, indicated that the vehicle/negative control mutant frequencies measured with the HIS49/CD59 method were less than half those measured with the CD45/ CD59 method (Fig. 8). It is not clear why this is the case; however, we can think of at least two potential explanations for the difference. First, the higher background frequencies with the CD45/CD59 method might be related to insufficient exclusion of CD45-negative cells in PB that were not RBCs and which have poor expression of CD59. Unlike the protocol that was used in the international Pig-a mutation assay trial (Dertinger et al., 2011c), our approaches did not use a density gradient to deplete nucleated leukocytes during the processing of samples. It is possible that positive selection for RBCs using HIS49/ CD59 might produce a sample more enriched for RBCs, and thus be better approach for measuring Pig-a mutant RBCs. Second, the combination of fluorescently conjugated antibodies might be responsible for the difference in background frequency. We used antibodies labeled with FITC and PECy5 or FITC and APC for the CD45/ CD59 method and the HIS49/CD59 method, respectively. APC is excited by the $635 \mathrm{~nm}$ laser, while both FITC and PECy5 are excited with the $488 \mathrm{~nm}$ laser. The data for HIS49/CD59 method were therefore acquired with two different lasers, which may have made it possible to discriminate the fluorescence with higher accuracy. Our analysis of the Pig- $a$ background mutant frequencies indicated that, for individual samples, mutant frequencies generated with the CD45/CD59 method were consistently higher than mutant frequencies generated using HIS49/CD59 method. Overall, the mean background mutant frequency generated with the HIS49/CD59 method was approx. $5 \times 10^{-6}$; we therefore consider that the HIS49/CD59 method is the better tool for measuring Pig- $a$ mutant RBCs, especially for compounds that have a weak mutagenic potential.

Further improvement to the sensitivity of the Pig-a mutant assay is possible through the assaying of more RBCs than were interrogated in this present study. Immunomagentic methods for depletion of wild-type RBCs (Dertinger et al., 2011b) or enrichment of RETs (Kimoto et al., 2011a) show promise in this regard. Dertinger et al. developed a system for depletion of CD59-positive RBCs 
via immunomagnetic column separation (Dertinger et al., 2011b). Their technique for enumerating Pig-a mutant frequencies is capable of interrogating more than $3 \times 10^{6}$ RETs and $1 \times 10^{8} \mathrm{RBCs}$ in a single sample. In addition, we have developed an assay for measuring Pig-a mutant cells in rat RETs, the PIGRET assay, that employs magnetic enrichment of CD71-positive RETs and enables us to interrogate more than $1 \times 10^{6} \mathrm{CD} 71 / \mathrm{HIS} 49$-positive RETs for Pig- $a$ mutation by FCM.

In conclusion, we have demonstrated that the RBC Pig- $a$ assay using anti-CD45 or HIS49 antibody was useful as a screening tool for evaluating in vivo mutagenicity. In particular, the protocol using positive selection for RBCs with HIS49 antibody might be better tool for detecting the mutagenic potential of chemicals due to its relatively low, stable background Pig-a mutant frequency. These results also suggest that the Pig- $a$ assay might be integrated into standard in vivo toxicology assays. More work is needed to define a standard Pig- $a$ assay protocol to efficiently evaluate the risk of in vivo mutagenicity.

\section{ACKNOWLEDGMENTS}

The authors would like to thank Dr. Robert H. Heflich for his critical review of this manuscript.

\section{REFERENCES}

Bryce, S.M., Bemis, J.C. and Dertinger, S.D. (2008): In vivo mutation assay based on the endogenous Pig-a locus. Environ. Mol. Mutagen., 49, 256-264.

Cammerer, Z., Bhalli, J.A., Cao, X., Coffing, S.L., Dickinson, D., Dobo, K.L., Dobrovolsky, V.N., Engel, M., Fiedler, R.D., Gunther, W.C., Heflich, R.H., Pearce, M.G., Shaddock, J.G., Shutsky, T., Thiffeault, C.J. and Schuler, M. (2011): Report on stage III Pig-a mutation assays using N-ethyl-N-nitrosoureacomparison with other in vivo genotoxicity endpoints. Environ. Mol. Mutagen., 52, 721-730.

Casciano, D.A., Aidoo, A., Chen, T., Mittelstaedt, R.A., Manjanatha, M.G. and Heflich, R.H. (1999): Hprt mutant frequency and molecular analysis of Hprt mutations in rats treated with mutagenic carcinogens. Mutat. Res., 431, 389-395.

Dertinger, S.D., Bryce, S.M., Phonethepswath, S. and Avlasevich, S.L. (2011b): When pigs fly: Immunomagnetic separation facilitates rapid determination of Pig-a mutant frequency by flow cytometric analysis. Mutat. Res., 721, 163-170.

Dertinger, S.D. and Heflich, R.H. (2011): In vivo assessment of Pig-a gene mutation-recent developments and assay validation. Environ. Mol. Mutagen., 52, 681-684.

Dertinger, S.D., Phonethepswath, S., Franklin, D., Weller, P., Torous, D.K., Bryce, S.M., Avlasevich, S., Bemis, J.C., Hyrien, O., Palis, J. and MacGregor, J.T. (2010): Integration of mutation and chromosomal damage endpoints into 28-day repeat dose toxicology studies. Toxicol. Sci., 115, 401-411.

Dertinger, S.D., Phonethepswath, S., Weller, P., Nicolette, J., Murray, J., Sonders, P., Vohr, H.W., Shi, J., Krsmanovic, L.,
Gleason, C., Custer, L., Henwood, A., Sweder, K., Stankowski, L.F.Jr., Roberts, D.J., Giddings, A., Kenny, J., Lynch, A.M., Defrain, C., Nesslany, F., van der Leede, B.J., Van Doninck, T., Schuermans, A., Tanaka, K., Hiwata, Y., Tajima, O., Wilde, E., Elhajouji, A., Gunther, W.C., Thiffeault, C.J., Shutsky, T.J., Fiedler, R.D., Kimoto, T., Bhalli, J.A., Heflich, R.H. and MacGregor, J.T. (2011c): International Pig-a gene mutation assay trial: evaluation of transferability across 14 laboratories. Environ. Mol. Mutagen., 52, 690-698.

Dertinger, S.D., Torous, D.K., Hayashi, M. and MacGregor, J.T. (2011a): Flow cytometric scoring of micronucleated erythrocytes: an efficient platform for assessing in vivo cytogenetic damage. Mutagenesis, 26, 139-145.

Dertinger, S.D., Torous, D.K. and Tometsko, K.R. (1996): Simple and reliable enumeration of micronucleated reticulocytes with a single-laser flow cytometer. Mutat. Res., 371, 283-292.

Dobo, K.L., Fiedler, R.D., Gunther, W.C., Thiffeault, C.J., Cammerer, Z., Coffing, S.L., Shutsky, T. and Schuler, M. (2011): Defining EMS and ENU dose-response relationships using the Pig-a mutation assay in rats. Mutat. Res., 725, 13-21.

Dobrovolsky, V.N., Boctor, S.Y., Twaddle, N.C., Doerge, D.R., Bishop, M.E., Manjanatha, M.G., Kimoto, T., Miura, D., Heflich, R.H. and Ferguson, S.A. (2010b): Flow cytometric detection of Pig-A mutant red blood cells using an erythroid-specific antibody: application of the method for evaluating the in vivo genotoxicity of methylphenidate in adolescent rats. Environ. Mol. Mutagen., 51, 138-145.

Dobrovolsky, V.N., Elespuru, R.K., Bigger, C.A., Robison, T.W. and Heflich, R.H. (2011): Monitoring humans for somatic mutation in the endogenous PIG-a gene using red blood cells. Environ. Mol. Mutagen., 52, 784-794.

Dobrovolsky, V.N., Miura, D., Heflich, R.H. and Dertinger, S.D. (2010a): The in vivo Pig-a gene mutation assay, a potential tool for regulatory safety assessment. Environ. Mol. Mutagen., 51, $825-835$.

Dobrovolsky, V.N., Shaddock, J.G., Mittelstaedt, R.A., Manjanatha, M.G., Miura, D., Uchikawa, M., Mattison, D.R. and Morris, S.M. (2009): Evaluation of Macaca mulatta as a model for genotoxicity studies. Mutat. Res., 673, 21-28.

Duesberg, P., Li, R., Fabarius, A. and Hehlmann, R. (2005): The chromosomal basis of cancer. Cell Oncol., 27, 293-318.

Gocke, E., Ballantyne, M., Whitwell, J. and Muller, L. (2009): MNT and MutaMouse studies to define the in vivo dose response relations of the genotoxicity of EMS and ENU. Toxicol. Lett., 190, 286-297.

Hamada, S., Sutou, S., Morita, T., Wakata, A., Asanami, S., Hosoya, S., Ozawa, S., Kondo, K., Nakajima, M., Shimada, H., Osawa, K., Kondo, Y., Asano, N., Sato, S., Tamura, H., Yajima, N., Marshall, R., Moore, C., Blakey, D.H., Schechtman, L.M., Weaver, J.L., Torous, D.K., Proudlock, R., Ito, S., Namiki, C. and Hayashi, M. (2001a): Evaluation of the rodent micronucleus assay by a 28-day treatment protocol: Summary of the 13 th Collaborative Study by the Collaborative Study Group for the Micronucleus Test (CSGMT)/Environmental Mutagen Society of Japan (JEMS)-Mammalian Mutagenicity Study Group (MMS). Environ. Mol. Mutagen., 37, 93-110.

Hamada, S., Yamasaki, K.I., Nakanishi, S., Omori, T., Serikawa, T. and Hayashi, M. (2001b): Evaluation of the general suitability of the rat for the micronucleus assay: the effect of cyclophosphamide in 14 strains. Mutat. Res., 495, 127-134.

Hartmann, A., Agurell, E., Beevers, C., Brendler-Schwaab, S., Burlinson, B., Clay, P., Collins, A., Smith, A., Speit, G., 
Rat Pig-a mutation assay for mutagenicity screening

Thybaud, V. and Tice, R.R. (2003): Recommendations for conducting the in vivo alkaline Comet assay. 4th International Comet Assay Workshop. Mutagenesis, 18, 45-51.

ICHS2(R1). Current step 4 version dated 9 November 2011. Guidance on genotoxicity testing and data interpretation for pharmaceuticals intended for human use. Available at www.ich.org.

Kawagoe, K., Takeda, J., Endo, Y. and Kinoshita, T. (1994): Molecular cloning of murine pig-a, a gene for GPI-anchor biosynthesis, and demonstration of interspecies conservation of its structure, function, and genetic locus. Genomics, 23, 566-574.

Kimoto, T., Chikura, S., Suzuki, K., Kobayashi, X., Itano, Y., Horibata, K., Honma, M., Dobrovolsky, V.N., Heflich, R.H., Miura, D. and Kasahara, Y. (2011a): Further development of the rat Pig-a mutation assay: measuring rat Pig-a mutant bone marrow erythroids and a high throughput assay for mutant peripheral blood reticulocytes. Environ. Mol. Mutagen., 52, 774-783.

Kimoto, T., Suzuki, K., Kobayashi, X.M., Dobrovolsky, V.N., Heflich, R.H., Miura, D. and Kasahara, Y. (2011b): Manifestation of Pig-a mutant bone marrow erythroids and peripheral blood erythrocytes in mice treated with N-ethyl-N-nitrosourea: direct sequencing of Pig-a cDNA from bone marrow cells negative for GPI-anchored protein expression. Mutat. Res., 723, 36-42.

Kirkland, D., Aardema, M., Henderson, L. and Muller, L. (2005): Evaluation of the ability of a battery of three in vitro genotoxicity tests to discriminate rodent carcinogens and non-carcinogens I. Sensitivity, specificity and relative predictivity. Mutat. Res., 584, 1-256.

Kondo, S. (1977): A test for mutation theory of cancer: carcinogenesis by misrepair of DNA damaged by 4-nitroquinoline 1-oxide. Br. J. Cancer, 35, 595-601.

Miura, D., Dobrovolsky, V.N., Kasahara, Y., Katsuura, Y. and Heflich, R.H. (2008a): Development of an in vivo gene mutation assay using the endogenous Pig-A gene: I. Flow cytometric detection of CD59-negative peripheral red blood cells and CD48-negative spleen T-cells from the rat. Environ. Mol. Mutagen., 49, 614-621.

Miura, D., Dobrovolsky, V.N., Kimoto, T., Kasahara, Y., Heflich, R.H. (2009): Accumulation and persistence of Pig-A mutant peripheral red blood cells following treatment of rats with single and split doses of N-ethyl-N-nitrosourea. Mutat. Res., 677, 86-92.

Miura, D., Dobrovolsky, V.N., Mittelstaedt, R.A., Kasahara, Y., Katsuura, Y. and Heflich, R.H. (2008b): Development of an in vivo gene mutation assay using the endogenous Pig-A gene: II. Selection of Pig-A mutant rat spleen T-cells with proaerolysin and sequencing Pig-A cDNA from the mutants. Environ. Mol. Mutagen., 49, 622-630.
Muller, L., Gocke, E., Lave, T. and Pfister, T. (2009): Ethyl methanesulfonate toxicity in Viracept--a comprehensive human risk assessment based on threshold data for genotoxicity. Toxicol. Lett., 190, 317-329.

Peruzzi, B., Araten, D.J., Notaro, R. and Luzzatto, L. (2010): The use of PIG-A as a sentinel gene for the study of the somatic mutation rate and of mutagenic agents in vivo. Mutat. Res., 705, 3-10.

Phonethepswath, S., Bryce, S.M., Bemis, J.C. and Dertinger, S.D. (2008): Erythrocyte-based Pig-a gene mutation assay: demonstration of cross-species potential. Mutat. Res., 657, 122-126.

Phonethepswath, S., Franklin, D., Torous, D.K., Bryce, S.M., Bemis, J.C., Raja, S., Avlasevich, S., Weller, P., Hyrien, O., Palis, J., Macgregor, J.T. and Dertinger, S.D. (2010): Pig-a mutation: kinetics in rat erythrocytes following exposure to five prototypical mutagens. Toxicol. Sci., 114, 59-70.

Rothfuss, A., Honma, M., Czich, A., Aardema, M.J., Burlinson, B., Galloway, S., Hamada, S., Kirkland, D., Heflich, R.H., Howe, J., Nakajima, M., O’Donovan, M., Plappert-Helbig, U., Priestley, C., Recio, L., Schuler, M., Uno, Y. and Martus, H.J. (2011): Improvement of in vivo genotoxicity assessment: combination of acute tests and integration into standard toxicity testing. Mutat. Res., 723, 108-120.

Stankowski, L.F.Jr., Roberts, D.J., Chen, H., Lawlor, T., McKeon, M., Murli, H., Thakur, A. and Xu, Y. (2011): Integration of Piga, micronucleus, chromosome aberration, and Comet assay endpoints in a 28-day rodent toxicity study with 4-nitroquinoline-1oxide. Environ. Mol. Mutagen., 52, 738-747.

Takeda, J., Miyata, T., Kawagoe, K., Iida, Y., Endo, Y., Fujita, T., Takahashi, M., Kitani, T. and Kinoshita, T. (1993): Deficiency of the GPI anchor caused by a somatic mutation of the PIG-A gene in paroxysmal nocturnal hemoglobinuria. Cell, 73, 703-711.

Thybaud, V., Dean, S., Nohmi, T., de Boer, J., Douglas, G.R., Glickman, B.W., Gorelick, N.J., Heddle, J.A., Heflich, R.H., Lambert, I., Martus, H.J., Mirsalis, J.C., Suzuki, T. and Yajima, N. (2003): In vivo transgenic mutation assays. Mutat. Res., 540, 141-151.

Tweats, D.J., Blakey, D., Heflich, R.H., Jacobs, A., Jacobsen, S.D., Morita, T., Nohmi, T., O'Donovan, M.R., Sasaki, Y.F., Sofuni, T., Tice, R. and IWGT Working Group (2007): Report of the IWGT working group on strategy/interpretation for regulatory in vivo tests II. Identification of in vivo-only positive compounds in the bone marrow micronucleus test. Mutat. Res., 627, 92-105.

Walker, D.M., Seilkop, S.K., Scott, B.R. and Walker, V.E. (2004): Hprt mutant frequencies in splenic T-cells of male F344 rats exposed by inhalation to propylene. Environ. Mol. Mutagen., 43, 265-272. 